\title{
Oral Cancer Knowledge, Attitudes, and Practices among Newly Graduated Dentists in Kuwait
}

\author{
Huda Shaker Nazar ${ }^{1 *}$, Jitendra Ariga ${ }^{2}$, Maddi Shyama ${ }^{1}$
}

\begin{abstract}
Background: Oral cancer awareness among newly graduated dentists could have a substantial impact in the prevention and early detection of oral cancer. Objective: This survey was undertaken to assess the knowledge, opinion, attitudes, and practices on oral cancer among newly graduated dentists in Kuwait. Methods: In this cross-sectional study, self-reported questionnaire was distributed to the newly graduated dentists. Of the 310 dentists who participated, $171(55.2 \%)$ were males and 139 (44.8\%) were females. The questionnaire included 23- questions on oral cancer knowledge, opinion, attitudes, and practices. Results: The mean age of the dentists was $25.8 \pm 2.4$ years and their mean years of experience $1.5 \pm 1.7$ years. Overall, a great majority of dentists $(95.8 \%)$ recognized tobacco use and alcohol consumption as very important risk factors for oral cancer occurrence. Almost all of participants were aware of the most common form of oral cancer (94.2\%). Most of the dentists correctly identified the most common site of oral cancer (93.5\%). Majority of the participants recognized the most commonly associated lesions with oral cancer $(91.6 \%)$. While $37.4 \%$ of the dentists agreed that they were adequately trained in oral cancer screening, most (89.7\%) believed that patients should have mandatory oral cancer screening at the clinics. Also, majority (95.8\%) expressed their willingness to attend continuing education and training courses in cancer screening and prevention. Most of the dentists $(81.9 \%)$ referred a patient with a suspicious lesion to a specialist. Conclusions: Majority of the dentists were aware and knowledgeable about various aspects of oral cancer. There is a need to emphasize and reinforce the training programs in oral cancer education mainly in prevention and early detection. Continuing education programs and workshops are highly recommended to raise awareness of the dentists on risk factors and diagnosis of oral cancer.
\end{abstract}

Keywords: Oral cancer- knowledge- opinion- attitude- practices- newly graduated dentists- Kuwait

Asian Pac J Cancer Prev, 23 (2), 459-465

\section{Introduction}

Oral cancer is a major public health problem which causes significant morbidity and mortality worldwide (Bray et al., 2018; Miranda-Filho and Bray, 2020; Sung et al., 2021). Early detection and prompt screening have revealed to be highly effective in decreasing the mortality and morbidity of oral cancer (Kujan and Sloan, 2013; Mariño et al., 2017). According to the World Health Assembly, oral health and overall health are related and several oral diseases are linked to non-communicable chronic diseases such as cardiovascular diseases, cancers and diabetes. The recommended preventive approach includes promotion of oral health within the family, schools, workplaces and comprises timely, comprehensive care within the primary health-care system (WHO, 2021). Primary prevention of oral cancer should essentially focus on the reduction of the main causes of cancer occurrence, specifically, tobacco smoking and alcohol consumption (Miranda-Filho and Bray, 2020). The main approaches incorporated by the World Health Organization (WHO)
Global Oral Health programs to prevent oral cancer are reduction of exposure to risk factors and early detection through screening (Petersen, 2009).

In 2020, according to global cancer statistics, oral cancer is the eighteenth most common cancer site accounting to $2 \%$ of new cases of all sites and $1.8 \%$ deaths (Sung et al., 2021). The incidence and mortality rates are higher in low and middle-income countries compared to high-income countries (Bray et al., 2018; Hung et al., 2020; Sung et al., 2021). In Kuwait, the age-standardized oral cancer incidence rate is 1.6 (per 100,000) and the mortality rate is 0.6 (Ferlay et al., 2008). In an earlier study of computer search in Kuwait cancer registry from 1979-1988, the nasopharynx and salivary glands were the main sites of head and neck cancers in Kuwait (Morris et al., 2000).

Previous studies in Kuwait, emphasized the requirement for improved knowledge and education for dentists on oral cancer (Joseph et al., 2012), and the necessity for an organized teaching program with importance on early recognition and risk factors among undergraduate 
dental students (Joseph et al., 2015). In a recent study conducted among dentists working at the primary oral health care centers in Kuwait, it was recommended that more continuing education programs on early detection of oral cancer and prevention of risk factors should be organized to train the dentists. This study emphasized that oral cancer screening should be a routine procedure for the high-risk patients in Kuwait (Nazar et al., 2019).

In Kuwait, the prevalence of smoking is $35 \%$ among males and 1.9\% among females (Memon et al., 2000). In a recent study, the prevalence of "ever smoker" in Kuwaiti men and women was $49.9 \%$ and $4.4 \%$, respectively, while the prevalence of "current smoker" was 39.2\% and 3.3\%, respectively (Alali et al., 2021). Another recent study in Kuwait revealed that a large percentage of adolescents were current users of e-cigarettes, conventional cigarettes, or hookah (Esmaeil et al., 2020). Early detection of oral cancer is required because of the high intake of tobacco products among Kuwaiti population. Also, implementing preventive measures through counselling by dentists along with the introduction of national policies for prevention of oral cancer should aid in reducing the future prevalence of smoking in Kuwait.

Dental professionals play a crucial role in the early detection of oral cancer through screening (Kujan and Sloan, 2013; Hashim et al., 2018). As they routinely examine the oral cavity; have the chance to diagnose oral cancer in asymptomatic patients before dissemination occurs to adjacent tissues (Fotedar et al., 2015; Pavão Spaulonci et al., 2018). Numerous studies have evaluated dental professionals' knowledge, attitudes and practices regarding oral cancer (Mariño et al., 2017; Haresaku et al., 2018; Hashim et al., 2018; Taheri et al., 2018; Jboor et al., 2019; Khattab et al., 2019; Leonel et al., 2019; Nazar et al., 2019; Aldossri et al., 2020; Alqutaibi et al., 2020; Kazmi et al., 2020; Algudaibi et al., 2021). In a recent systematic review study, many oral health care professionals worldwide felt the necessity to enhance their knowledge, attitude, and practices in oral cancer awareness and emphasized the necessity for an effective pre- and post-graduation training (Coppola et al., 2021).

Although, oral cancer awareness among newly graduated dentists could have a substantial impact in the prevention and early detection of oral cancer, little is known about their awareness. A small number of earlier studies have shown that newly graduated dentists had more knowledge in comparison to senior dental professionals (Alaizari and Al-Maweri, 2014; Eltelety et al., 2014; Pavão Spaulonci et al., 2018). Recently graduated dentists in Saudi Arabia, had higher level of knowledge about risk factors, while those who graduated earlier had higher overall knowledge (Eltelety et al., 2014). Also, recently graduated dentists in Yemen had better knowledge on diagnosis and clinical practices in comparison to dentists who had graduated earlier (Alaizari and Al-Maweri, 2014). Furthermore, in a recent study in Brazil, newly graduated dentists had higher knowledge level in comparison to senior dentists who had more practice experience (Pavão Spaulonci et al., 2018).

No information is available regarding the oral cancer awareness among newly graduated dentists in Kuwait.
Hence, it is important to know their level of knowledge, behaviors, and practices as they will be joining the primary oral health care clinics, wherein they will be delivering oral health care for patients. Also, the evaluation of oral cancer knowledge, attitude, and practices among them is of great importance and the information obtained can aid to assess the necessity to implement public policies aimed at continued education for oral cancer. This survey was undertaken to assess the current knowledge, opinions, attitudes, and practices on oral cancer among newly graduated dentists in Kuwait.

\section{Materials and Methods}

\section{Study design}

This cross-sectional study was conducted among the newly graduated dentists, Ministry of Health in Kuwait. The study protocol was approved by the Ethical Research Committee of School Oral Health Program (SOHP), Kuwait-Forsyth in 2017. This study was conducted in accordance with the laws of the State of Kuwait, rules and regulations of the Ministry of Health, and was in full accordance with the World Medical Association Declaration of Helsinki.

\section{Study setting and participants}

Only the newly graduated dentists working in the Ministry of Health, Kuwait who attended the SOHP continuing education courses during 2017-2018 were included in this study. The dentists were working at the polyclinics, specialty dental centers, and the SOHP clinics as a part of their clinical training rotation. The continuing education course is a part of the training program after graduation in Kuwait. The questionnaire was distributed at the auditorium before the presentation on early detection of oral cancer by visual examination. Overall, 310 dentists received the questionnaire and participated in this study.

\section{Study questionnaire}

A self-reported 23-item questionnaire in English was designed and used in this study, which included previously validated questions and also questions designed for this study (Patton et al., 2005; Gajendra et al., 2006; Nazar et al., 2019). The first section of the questionnaire included 6 questions regarding dentists' demographic characteristics. The demographic data collected were age, gender, nationality, level of education, years of experience, and the working site. The second section of the questionnaire included 17 questions to assess the dentists' oral cancer knowledge, opinions, attitude, and practices. Nine knowledge questions were about the oral cancer risk factors, most common forms of oral cancer, common age of diagnosis, most common sites of oral cancer, symptoms of both early, late stage of oral cancer, and the features of oral cancer metastases. Three questions were about the dentists' opinion on oral cancer training, overall dentists' current knowledge, and mandatory oral cancer screening. One attitude question was regarding attending oral cancer continuing education courses. Four questions were about their current practices including evaluating oral cancer risk factors and managing patients with suspicious lesions. 
DOI:10.31557/APJCP.2022.23.2.459

Oral Cancer Knowledge, Attitudes and Practices

\section{Statistical analysis}

Data were analyzed using Epi-Info 3.5.3 and SPSS, windows version 22.0. Frequencies and means (SD) were used for data description. Chi-square test was utilized to compare differences between the responses according to gender, nationality, and level of education. An independent-samples t-test and One-way Analysis of Variance (ANOVA) were used to examine the difference in mean knowledge, attitude, and practice scores according to gender, nationality, level of education, age and years of experience. The significance level used was $\mathrm{p}<0.05$.

\section{Results}

Among the 310 questionnaires distributed, all the dentists answered and completed the questionnaire, resulting in a $100 \%$ response rate. Of the 310 dentists, $171(55.2 \%)$ were males and $139(44.8 \%)$ were females. Majority 298 (96.1\%) were Kuwaiti, and only 12 (3.9\%) were from other nationalities. The mean age was 25.8 \pm 2.4 years. Majority of the dentists 293 (94.5\%) had a bachelor degree, while 17 (5.5\%) had more than bachelor degree $(2.6 \%$ master degree, $1.9 \%$ MFDS, $0.3 \%$ MEGD. $0.6 \% \mathrm{PhD}$ ). Their mean years of experience was $1.5 \pm 1.7$ years. Table 1 summarizes the demographic characteristics of the participants.

Majority of the dentists $253(81.5 \%)$ correctly answered the knowledge questions. Overall, most of dentists $(95.8 \%)$ recognized tobacco use and alcohol consumption as the major risk factors for oral cancer. Almost all were aware of the most common form of oral cancer (94.2\%). Approximately, all female dentists (97.8\%) were aware of the most common form of oral cancer when compared to the male dentists $(91.2 \%)$ (p $=0.010)$. Most of the dentists correctly identified the most common site of oral cancer $(93.5 \%)$ and the most likely lesions associated with oral cancer (91.6\%). A great number of dentists $(82.9 \%)$ recognized the features of oral cancer metastases. Significantly, more female dentists $(90.6 \%)$ identified correctly the lymph node characteristics of oral cancer metastases when compared to male dentists $(76.6 \%)(\mathrm{p}=0.001)$. All the dentists with a master degree identified correctly the features of cancer

Table 1. Demographic Characteristics of the Participating Dentists $(\mathrm{N}=310)$

\begin{tabular}{lc}
\hline Variable & No. $(\%)$ \\
\hline Gender & $171(55.2)$ \\
$\quad$ Male & $139(44.8)$ \\
$\quad$ Female & $25.8 \pm 2.4$ \\
Age in years (mean \pm SD) & \\
Nationality & $298(96.1)$ \\
$\quad$ Kuwaiti & $12(3.9)$ \\
$\quad$ Non-Kuwaiti $($ Others $)$ & \\
Level of education & $293(94.5)$ \\
$\quad$ Bachelor degree & $17(5.5)$ \\
$\quad$ More than Bachelor degree & $1.5 \pm 1.7$ \\
Years of experience (mean \pm SD) & \\
\hline
\end{tabular}

Table 2. Descriptive Analyses of Dentists' Knowledge

\begin{tabular}{lccc}
\hline Question & $\begin{array}{c}\text { Males } \\
\text { No. (\%) }\end{array}$ & $\begin{array}{c}\text { Females } \\
\text { No. (\%) }\end{array}$ & $\begin{array}{c}\text { Overall } \\
\text { No. (\%) }\end{array}$ \\
\hline Most common risk factors of oral cancer \\
Correct & $162(94.7)$ & $135(97.1)$ & $297(95.8)$ \\
Incorrect & $9(5.3)$ & $4(2.9)$ & $13(4.2)$ \\
Most likely lesions associated with oral cancer & \\
Correct & $156(91.2)$ & $128(92.1)$ & $284(91.6)$ \\
Incorrect & $15(8.8)$ & $11(7.9)$ & $26(8.4)$
\end{tabular}

Conditions which are associated with fibrosis and reduced mouth opening

$\begin{array}{lccc}\text { Correct } & 154(90.1) & 130(93.5) & 284(91.6) \\ \text { Incorrect } & 17(9.9) & 9(6.5) & 26(8.4)\end{array}$

Symptoms of the late stage of oral cancer

$\begin{array}{lccc}\text { Correct } & 156(91.2) & 128(92.1) & 284(91.6) \\ \text { Incorrect } & 15(8.8) & 11(7.9) & 26(8.4)\end{array}$

Most common form of oral cancer

$\begin{array}{lccc}\text { Correct } & 156(91.2) & 136(97.8) & 292(94.2) \\ \text { Incorrect } & 15(8.8) & 3(2.2) & 18(5.8)\end{array}$

Most common site of oral cancer

$$
\begin{array}{lccc}
\text { Correct } & 158(92.4) & 132(95.0) & 290(93.5) \\
\text { Incorrect } & 13(7.6) & 7(5.0) & 20(6.5)
\end{array}
$$

Lymph node characteristics of oral cancer metastases

$\begin{array}{lccc}\text { Correct } & 131(76.6) & 126(90.6) & 257(82.9) \\ \text { Incorrect } & 40(23.4) & 13(9.4) & 53(17.1)\end{array}$

Majority of oral cancers diagnosed at age
Correct
$73(42.7)$
55 (39.6)
$128(41.3)$
Incorrect
$98(57.3) \quad 84(60.4)$
$182(58.7)$

Symptoms most commonly expressed in early oral cancer

\begin{tabular}{llll} 
Correct & $86(50.3)$ & $71(51.1)$ & $157(50.6)$ \\
Incorrect & $85(49.7)$ & $68(48.9)$ & $153(49.4)$ \\
\hline
\end{tabular}

metastases when compared to those with bachelor degree $(81.9 \%)(p=0.038)$. Majority of dentists $(91.6 \%)$ reported

Table 3. Descriptive Analyses of Dentists' Opinion and Attitude

\begin{tabular}{lccc}
\hline Question & Males & Females & Overall \\
& No. (\%) & No. (\%) & No. $(\%)$ \\
\hline \multicolumn{4}{c}{ Think patient should have mandatory oral cancer screening } \\
Yes & $154(90.1)$ & $124(89.2)$ & $278(89.7)$ \\
No & $17(9.9)$ & $15(10.8)$ & $32(10.3)$ \\
Knowledge about oral cancer is current \\
Yes & $81(47.4)$ & $86(61.9)$ & $167(53.9)$ \\
No & $90(52.6)$ & $53(38.1)$ & $143(46.1)$ \\
Adequate training to perform an oral cancer screening \\
Yes & $68(39.8)$ & $48(34.5)$ & $116(37.4)$ \\
No & $103(60.2)$ & $91(61.5)$ & $194(62.6)$ \\
Interested in continuing education courses & regarding oral \\
cancer & \multicolumn{4}{c}{} \\
Yes & $163(95.3)$ & $134(96.4)$ & $297(95.8)$ \\
No & $8(4.7)$ & $5(3.6)$ & $13(4.2)$ \\
\hline
\end{tabular}

Asian Pacific Journal of Cancer Prevention, Vol 23 
Table 4. Descriptive Analyses of Dentists' Practice

\begin{tabular}{lccc}
\hline Question & Males & Females & Overall \\
& No. (\%) & No. (\%) & No. (\%) \\
\hline Refer patient with suspicious lesion to a specialist \\
Always & $92(53.8)$ & $85(61.2)$ & $188(57.1)$ \\
Usually & $42(24.6)$ & $35(25.2)$ & $77(24.8)$ \\
Sometimes & $25(14.6)$ & $16(11.5)$ & $41(13.2)$ \\
Never & $12(7.0)$ & $3(2.2)$ & $15(4.8)$ \\
Review patients' risk factors of oral cancer & \\
Always & $19(11.1)$ & $21(15.1)$ & $40(12.9)$ \\
Usually & $36(21.1)$ & $45(32.4)$ & $81(26.1)$ \\
Sometimes & $98(57.3)$ & $85(46.8)$ & $163(52.6)$ \\
Never & $18(10.5)$ & $8(5.8)$ & $26(8.4)$ \\
Ask patient about tobacco use & & \\
Always & $81(47.4)$ & $84(60.4)$ & $165(53.2)$ \\
Usually & $51(29.8)$ & $34(24.5)$ & $85(27.4)$ \\
Sometimes & $37(21.6)$ & $16(11.5)$ & $53(17.1)$ \\
Never & $2(1.2)$ & $5(3.6)$ & $7(2.3)$ \\
Ask patient about alcohol consumption & \\
Always & $17(9.9)$ & $10(7.2)$ & $27(8.7)$ \\
Usually & $22(12.9)$ & $25(18.0)$ & $47(15.2)$ \\
Sometimes & $79(46.2)$ & $60(43.2)$ & $139(44.8)$ \\
Never & $53(31.0)$ & $44(31.7)$ & $97(31.3)$ \\
\hline \multicolumn{4}{c}{}
\end{tabular}

the symptoms of late stage of oral cancer correctly, and half $(50.6 \%)$ were aware of the symptoms of the early stage of oral cancer. Significantly, greater number of Kuwaiti dentists (93\%) reported symptoms of late stage of oral cancer correctly when compared to non-Kuwaiti dentists $(58.5 \%)(p=0.001)$. Less than half $(41.3 \%)$ of dentists knew the most common age of oral cancer diagnosis. Descriptive analysis of knowledge responses is summarized in Table 2. The mean knowledge score was $7.33 \pm 1.12$. Female dentists had significantly better mean knowledge scores when compared to males ( $7.49 \mathrm{vs}$. $7.20, \mathrm{p}=0.027)$. There was no significant difference in knowledge based on age, nationality, or level of education.

Overall, more than half $(60.3 \%)$ of the dentists answered opinion questions positively. While $37.4 \%$ agreed that they were adequately trained in oral cancer screening, the majority $(89.7 \%)$ believed that patients should have mandatory screening at the clinics. Half of the dentists $(53.9 \%)$ indicated that their oral cancer knowledge is current. More female dentists (61.9\%) reported positively as their oral cancer knowledge as current when compared to less than half of the male dentists $(47.4 \%)(p=0.007)$. The mean opinion score of the dentists was $1.81 \pm 0.84$. Dentists with a master degree had higher mean opinion score compared to those with only a bachelor degree ( 2.35 vs. $1.78, \mathrm{p}=0.006)$. Opinion responses are summarized in Table 3 . A vast majority (95.8\%) expressed their willingness to attend continuing education and training courses in cancer screening and prevention, with a mean score of $0.96 \pm 0.20$. Overall, more than half $(56.4 \%)$ of dentists followed the ideal practice regarding screening and managing oral cancer. Most of the dentists $(81.9 \%)$ referred a patient with a suspicious lesion to a specialist. More than one-third (39\%) reviewed their patients' oral cancer risk factors. Nearly half of the female dentists $(47.5 \%)$ reviewed oral cancer risk factors when compared to one-third of the male dentists $(32.2 \%)(p=0.038)$. Majority of the dentists $(80.6 \%)$ reported that they assessed the use of tobacco in their practice. Significantly, greater number of female practitioners (84.9\%) assessed use of tobacco when compared to male practitioners $(77.2 \%)(p=0.020)$. Only a quarter of the dentists $(24.0 \%)$ asked their patients about alcohol consumption. The mean practice score was $11.11 \pm 2.42$. There was significant difference in practice scores based on gender $(p=0.026)$, and the level of education $(p=0.002)$. Distribution of different practice responses is summarized in Table 4.

\section{Discussion}

Oral cancer knowledge and awareness of dentists is of vital importance as they are usually the first point of contact to patients for oral health care, who can emphasize on prevention and also for early detection which can eventually lower the occurrence of oral cancer (Jboor et al., 2019; Nazar et al., 2019). No information is available regarding the oral cancer awareness among newly graduated dentists in Kuwait. In this study, newly graduated dentists' knowledge, attitude, and practices regarding oral cancer was assessed in Kuwait.

In the current study, $(81.5 \%)$ of the newly graduated dentists had good knowledge on oral cancer, which was higher than in a previous study, wherein two-thirds (65\%) of dentists in Sri Lanka had adequate knowledge of oral cancer screening (Ariyawardana and Ekanayake 2008). However, in a prior study, only $37.5 \%$ of the senior dental students in Iran, had a good knowledge on oral cancer, their overall knowledge was not adequate and required additional training and education (Honarmand et al., 2014).

In our study, majority of the participants $(95.8 \%)$ identified smoking and alcohol as very important risk factors for oral cancer occurrence. This high level of practitioners' knowledge about oral cancer risk factors coincided with many earlier studies and similarly in previous studies, majority of dentists stated tobacco use and alcohol consumption as the main risk factors for oral cancer occurrence (Mariño et al., 2017; Hashim et al., 2018; Haresaku et al., 2018; Kebabcığlu and Pekiner 2018; Pavão Spaulonci et al., 2018; Keser and Pekiner 2019; Leonel et al., 2019; Jboor et al., 2019; Nazar et al., 2019; Aldossri et al., 2020; Algudaibi et al., 2021). Also, in a recent study in Kuwait, majority of the undergraduate dental students and medical students stated that tobacco smoking has a significant effect for the occurrence of oral cancer (Saleem et al., 2021). In the same study, all of the dental students $(100 \%)$ and $73.8 \%$ of the medical students recognized betel quid and smokeless tobacco as risk factors associated in the progression of oral cancer (Saleem et al., 2021). There was no significant difference 
between the responses of the junior and senior dental clinicians in Brazil regarding the oral cancer risk factors (Pavão Spaulonci et al., 2018). Comparable oral cancer risk factors were noted by majority of dentists (Joseph et al., 2012; Jboor et al., 2019; Khattab et al., 2019; Algudaibi et al., 2021). However, alcohol was poorly identified as a risk factor for oral cancer among Japanese oral health professionals (Haresaku et al., 2018).

In this study, almost all participants $(94.2 \%)$ were aware of the most common form of oral cancer. Another study among the general dental practitioners in Iran, revealed that awareness of most common form of cancer was $80 \%$, and more than half $(57.5 \%)$ of dentists correctly responded questions concerning the most common type of precancerous lesion (Taheri et al., 2018). In a recent survey, two-thirds $(66.2 \%)$ of the surveyed dentists in Brazil pointed out that squamous cell carcinoma was the most common type of oral cancer, while, one-third (33.8\%) of the participants were unaware of the most common form of oral cancer (Leonel et al 2019). Another study revealed that $18.4 \%$ of dentists in Iran were unaware of the most common type of oral cancer (Mehdizadeh et al., 2014).

Majority of the recently graduated dentists in this study correctly recognized the most common site of oral cancer (93.5\%), when compared to previous studies (Hashim et al 2018; Pavão Spaulonci et al., 2018; Leonel et al., 2019; Jboor et al., 2019). Similarly, in a recent study among undergraduate University students in Kuwait, significantly more dental students $(98.5 \%)$ were able to detect the lateral border of the tongue as a high-risk anatomical location for the occurrence of oral cancer compared to about one-third (35.4\%) of the medical students (Saleem et al., 2021). In an earlier study, only a few dentists in Colombia knew the most frequent location of oral malignancy (Rocha-Buelvas et al. 2012). Only 55.5\% of dentists in Brazil knew the most frequent anatomical region for oral cancer (Pavão Spaulonci et al., 2018). More than two-thirds (71.9\%) of the primary health care dentists in Brazil indicated that the tongue and floor of the mouth were the most frequent sites of oral cancer (Leonel et al., 2019). Majority of dental students $(79.3 \%)$ in Spain stated that they routinely examine the oral mucosa of their patients (Frola and Barrios, 2017).

The present study showed that majority of participants $(91.6 \%)$ identified the most commonly associated lesions with oral cancer, as observed in another survey among Brazilian dentists, wherein $85.9 \%$ identified leukoplakia as the likely lesion condition associated with oral cancer (Leonel et al., 2019). Also, majority (98.5\%) of dental students and $70.8 \%$ of medical students in Kuwait determined that erythroleukoplakia has the highest premalignant potential to develop into oral cancer (Saleem et al., 2021).

Our study revealed that majority of dentists $(82.9 \%)$ knew about the features of oral cancer metastases and identified correctly the lymph node characteristics. This was higher than among earlier studies; wherein less than $40 \%$ of dentists stated that they practiced lymph node palpation during screening of their patients (Kebabcioğlu and Pekiner 2018). In a study among dentists in Brazil, one-third of them did not know about regional oral cancer metastases and only two-thirds of them knew about oral cancer metastases (Pavão Spaulonci et al., 2018). In a study among undergraduate students in Kuwait, more than two-thirds (70\%) of the dental students and majority $(81.5 \%)$ of the medical students considered the cervical lymph nodes to be the most frequent site of metastases (Saleem et al., 2021).

In this study, almost all of participants were aware of the symptoms of late stage of oral cancer, and half were aware of the symptoms of the early stage of oral cancer. Similar to this study, $54.9 \%$ of dentists in Iran correctly replied the most common symptoms of cancerous lesions (Taheri at al 2018). Majority of the dental students (98.5\%) and medical students $(83.1 \%)$ in Kuwait answered that a non-healing ulcer for more than three weeks to be a warning feature of oral cancer (Saleem et al., 2021). In this study, more than one-third $(37.4 \%)$ of the dentists were of opinion that they were adequately trained in oral cancer screening; however, majority $(89.7 \%)$ believed that patients' should have mandatory oral cancer screening at the clinics. Similar to this study, less than $40 \%$ of Japanese oral health professionals stated that they performed oral cancer screenings in their patients (Haresaku et al., 2018). However, a vast majority, (90\%) of the professionals in Japan indicated that they required supplementary training in their oral cancer practices (Haresaku et al., 2018). In a recent systematic review study, globally, the frequency of oral squamous cell carcinoma screening by oral health care providers was low (Coppola et al., 2021).

In the present study, more than half $(53.9 \%)$ of dentists reported their opinion as their knowledge about oral cancer was up-to-date. Higher percentages (68-70\%) of dentists in Sri Lanka indicated that their oral cancer/ pre cancer knowledge was current (Ariyawardana and Ekanayake 2008). In this study, majority of the dentists $(81.9 \%)$ would refer a patient with a suspicious lesion to a specialist. Similarly, in an earlier study $72 \%$ of dentists in Sri Lanka agreed to refer a patient if a suspicious lesion was found. In the same study, nearly $81 \%$ agreed that they were sufficiently trained in oral cancer screening while $70 \%$ felt they required more training (Ariyawardana and Ekanayake 2008).

There is a requirement for regular continuing education programs for apprising the knowledge level of dental professionals (Tadbir et al., 2013). Also, there is a necessity for intensive training and workshops to increase the awareness and abilities to diagnose oral cancer (Kazmi et al., 2020). In this study, most of the dentists (95.8\%) showed a keen interest to attend continuing education courses in oral cancer prevention and screening, as was previously mentioned in other studies (Saleh et al., 2014; Leonel et al 2019). However, in a recent study, half $(50.7 \%)$ of the primary health care dentists in Brazil stated that they did not enroll to any continued education program for more than two years (Leonel et al., 2019).

The strength of this study, is that this is the first study in Kuwait among newly graduated dentists assessing their knowledge, attitude and practices on oral cancer. The survey questionnaire was distributed before a lecture presentation on "oral cancer visual screening", as a result, 
all the participated dentists who graduated from different dental schools would act as an advocate to their patients in the primary oral health clinics as well as in the specialty dental centers. Another strength of the current study, is the complete response rate among the participating dentists. The limitation is that, this survey included only the newly graduated dentists who attended the SOHP continuing education lectures. Although the attendee dentists may not be a representative sample of dentists, the results from this survey provide a valuable estimate of the oral cancer knowledge, attitude and practices in Kuwait.

In conclusion, a vast majority of dentists in this study were well aware and knowledgeable about different aspects of oral cancer. There is a need to emphasize and reinforce the training programs in oral cancer education mainly in prevention and early detection. Continuing education programs and workshops are highly recommended to raise the awareness of the dentists on risk factors and diagnosis of oral cancer.

\section{Author Contribution Statement}

HN: Study design, planning, conduction, supervision administration, data collection, analysis interpretation, manuscript writing, and revision. JA: Study supervision, manuscript writing and revision. MS: Data analysis, interpretation, manuscript writing, revision. All the authors reviewed, and approved the manuscript.

\section{Acknowledgements}

We express our appreciation to all the dentists who participated in the study.

\section{Ethical statement}

The study protocol was approved by the Ethical Research Committee of School Oral Health Program, Kuwait-Forsyth on 18th, January 2017. This study was conducted in accordance with the laws of the State of Kuwait, rules and regulations of the Ministry of Health, and was in full accordance with the World Medical Association Declaration of Helsinki.

Availability of data

The data that support the findings of this study are available on request from the corresponding author.

\section{Conflict of interest}

The authors declare no conflict of interest regarding the study.

\section{References}

Alaizari NA, Al-Maweri SA (2014). Oral cancer: knowledge, practices and opinions of dentists in Yemen. Asian Pac J Cancer Prev, 15, 5627-31.

Alali WQ, Longenecker JC, Alwotyan R, et al (2021). Prevalence of smoking in the Kuwaiti adult population in 2014: a crosssectional study. Environ Sci Pollut Res, 28, 10053-67.

Aldossri M, Okoronkwo C, Dodd V, Manson H, Singhal S (2020). Dentists' capacity to mitigate the burden of oral cancers in Ontario, Canada. J Can Dent Assoc, 86, k2.

Algudaibi LY, AlMeaigel S, AlQahtani N, Shaheen NA,
Aboalela A (2021). Oral and oropharyngeal cancer: Knowledge, attitude and practices among medical and dental practitioners. Cancer Rep (Hoboken), M3, e1349.

Alqutaibi AY, Borzangy S, Al-Maweri SA, Aboalrejal A (2020). Early detection of oral cancer and potentially malignant disorders: Experiences, practices, and beliefs of prosthodontists practicing in Saudi Arabia. J Prosthet Dent, 26, S0022-3913(20)30434-0.

Ariyawardana A, Ekanayake L (2008). Screening for oral cancer/ precancer: knowledge and opinions of dentists employed in the public sector dental services of Sri Lanka. Asian Pac J Cancer Prev, 9, 615-8.

Bray F, Ferlay J, Soerjomataram I, et al (2018). Global cancer statistics 2018: GLOBOCAN estimates of incidence and mortality worldwide for 36 cancers in 185 countries. $C A$ Cancer J Clin, 68, 394-424.

Coppola N, Mignogna MD, Rivieccio I, et al (2021). Current knowledge, attitudes, and practice among health care providers in OSCC awareness: Systematic review and metaanalysis. Int J Environ Res Public Health, 18, 4506.

Eltelety SMK, Hassan MHA, El Kassimi F, El qahatani N, Mohamed N (2014). Knowledge, attitudes and practices regarding oral cancer among dentists in Jeddah. Cairo Dent $J, 30,1-17$.

Esmaeil A, Alshammasi A, Almutairi W, et al (2020). Patterns of electronic cigarette, conventional cigarette, and hookah use and related passive exposure among adolescents in Kuwait: a cross-sectional study. Tob Induc Dis, 18, 59.

Ferlay J, Shin HR, Bray F, et al (2010). GLOBOCAN 2008, Cancer incidence and mortality wordwide: IARC CancerBase No 10. Lyon, International Agency for Research on Cancer, http://globocan.iarc.fr.

Fotedar S, Bhardwaj V, Manchanda K, et al (2015). Knowledge, attitude and practices about oral cancers among dental students in HP Government Dental College, ShimlaHimachal Pradesh. South Asian J Cancer, 4, 65-7.

Frola MI, Barrios R (2017). Knowledge and attitudes about oral cancer among dental students after Bologna Plan implementation. J Cancer Educ, 32, 634-9.

Gajendra S, Cruz G, Kumar J (2006). Oral cancer prevention and early detection: knowledge, practices, and opinions of oral health care providers in New York State. $J$ Cancer Educ, 21, 157-62.

Hashim R, Abo-Fanas A, Al-Tak A, Al-Kadri A, Abu Ebaid Y (2018). Early detection of oral cancer- dentists' knowledge and practices in the United Arab Emirates. Asian Pac J Cancer Prev, 19, 2351-5.

Haresaku S, Makino M, Sugiyama S, Naito T, Mariño RJ (2018). Comparison of practices, knowledge, confidence, and attitude toward oral cancer among oral health professionals between Japan and Australia. J Cancer Educ, 33, 429-35.

Honarmand M, Hajihosseini A, Akbari F (2014). Oral cancer knowledge of senior dental students in Zahedan, South-East of Iran. Asian Pac J Cancer Prev, 15, 3017-20.

Hung LC, Kung PT, Lung CH, et al (2020). Assessment of the risk of oral cancer incidence in a high-risk population and establishment of a predictive model for oral cancer incidence using a population-based cohort in Taiwan. Int J Environ Res Public Health, 17, 665.

Jboor DH, Al-Darwish MS, Nur U (2019). Oral cancer knowledge, attitude and practice of dentists in the State of Qatar. Dent J, 7, 43.

Joseph BK, Sundaram DB, Sharma P (2012). Oral cancer awareness among dentists in Kuwait. Med Princ Pract, 21, 164-70.

Joseph BK, Sundaram DB, Ellepola AN (2015). Assessing oral cancer knowledge among undergraduate dental students in 
Kuwait University. J Cancer Educ, 30, 415-20.

Kazmi F, Alkait S, Alghamdi H, Alhussain G, Tabassum A (2020). Assessing knowledge, attitude and practices for oral squamous cell carcinoma among health care professionals in Princess Nourah University, Riyadh, KSA. Asian Pac J Cancer Prev, 21, 539-45.

Kebabcioğlu Ö, Pekiner FN (2018). Assessing oral cancer awareness among dentists. $J$ Cancer Educ, 33, 1020-6.

Keser G, Pekiner FN (2019). Assessing Oral Cancer Awareness Among Dental Students. J Cancer Educ, 34, 512-8.

Khattab NMA, Elheeny AAH, Tony GA (2019). Oral-cancer knowledge, practice, and attitude assessment of dentists in Upper Egypt: A cross-sectional study. Clin Exp Dent Res, $\mathbf{5}, 121-7$.

Kujan O, Sloan P (2013). Dilemmas of oral cancer screening: an update. Asian Pac J Cancer Prev, 14, 3369-73.

Leonel ACLDS, Soares CBRB, Lisboa de Castro JF, et al (2019). Knowledge and attitudes of primary health care dentists regarding oral cancer in Brazil. Acta Stomatol Croat, 53, 55- 63.

Mariño R, Haresaku S, McGrath R, et al (2017). Oral cancer screening practices of oral health professionals in Australia. BMC Oral Health, 17, 151 .

Mehdizadeh M, Majidi MS, Sadeghi S, Hamzeh M (2014). Evaluation of knowledge, attitude and practice of general dentists regarding oral cancer in Sari, Iran. Iran J Cancer Prev, 7, 101-4.

Memon A, Moody PM, Sugathan TN, et al (2000). Epidemiology of smoking among Kuwaiti adults: prevalence, characteristics, and attitudes. Bull World Health Organ, 78, 1306-10.

Miranda-Filho A, Bray F (2020). Global patterns and trends in cancers of the lip, tongue and mouth. Oral Oncol, 102, 104551.

Morris RE, Mahmeed BE, Gjorgov AN, Jazzaf HG, Rashid BE (2000). The epidemiology of lip, oral cavity and pharyngeal cancers in Kuwait. Br J Oral Maxillofac Surg, 38, 316-9.

Nazar H, Shyama M, Ariga J, et al (2019). Oral cancer knowledge, attitudes and practices among primary oral health care dentists in Kuwait. Asian Pac J Cancer Prev, 20, 1531-6.

Patton LL, Elter JR, Southerland JH, Strauss RP (2005). Knowledge of oral cancer risk factors and diagnostic concepts among North Carolina dentists. Implications for diagnosis and referral. J Am Dent Assoc, 136, 602-10.

Pavão Spaulonci G, Salgado de Souza R, Gallego Arias Pecorari V, Lauria Dib L (2018). Oral cancer knowledge assessment: newly graduated versus senior dental clinicians. Int J Dent, 14, 9368918.

Petersen PE (2009). Oral cancer prevention and control - the approach of the World Health Organization. Oral Oncol, 45, 454-60.

Rocha-Buelvas A, Hidalgo-Patiño C, Collela G, Angelillo I (2012). Oral cancer and dentists: knowledge, attitudes and practices in a South Colombian context. Acta Odontol Latinoam, 25, 155-62.

Saleem L, Mahmoud H, Joseph B (2021). Knowledge and attitude about oral cancer among medical and dental students at Kuwait University: A cross-sectional study. Asian Pac J Cancer Care, 6, 277-83.

Saleh A, Kong YH, Vengu N, et al (2014). Dentists' perception of the role they play in early detection of oral cancer. Asian Pac J Cancer Prev, 15, 229-37.

Sung H, Ferlay J, Siegel RL, et al (2021). Global cancer statistics 2020: GLOBOCAN estimates of incidence and mortality worldwide for 36 cancers in 185 countries. CA Cancer J Clin, 4.

Tadbir AA, Ebrahimi H, Pourshahidi S, Zeraatkar M (2013).
Evaluation of levels of knowledge about etiology and symptoms of oral cancer in southern Iran. Asian Pac J Cancer Prev, 14, 2217-20.

Taheri JB, Namazi Z, Azimi S, et al (2017). Knowledge of oral precancerous lesions considering years since graduation among dentists in the Capital city of Iran: a pathway to early oral cancer diagnosis and referral?. Asian Pac J Cancer Prev, 24, 2103-8.

World Health Organization (2021). World Health Assembly Resolution paves the way for better oral health care. https:// www.who.int/news/item/27-05-2021.

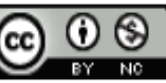

This work is licensed under a Creative Commons AttributionNon Commercial 4.0 International License. 